THURSDAY, FEBRUARY 15,1872

\section{THE POSITION OF THE CENTRE OF GRAVITY IN INSECTS}

M $\mathrm{Y}$ researches on the conditions of equilibrium in 1 living beings, have led me to the conclusion that a complete knowledge of them is only possible when the position of the centre of gravity in each is known.

At present the knowledge of the mechanism of the Articulata has made considerable progress, thanks to the use of processes of investigation borrowed from Physics; and it appeared to me, that there would be real utility in the description of an easy method for the discovery of the centre of gravity in the Articulata, and the results which its application to insects has allowed me to obtain. I am, unfortunately, unable in a simple részuné to give a description of the instrument which I have employed. A very short description without an engraving is necessarily obscure, and loses all utility. I shall simply say that the instrument in question is a reproduction, on a small scale and with some improvements, of that which Barelli has invented for the determination of the centre of gravity in man. And with regard to the results of my experiments, I must also renounce the idea of giving them in the form they assumed in my work, that is, without the considerable number of figures combined in tables. I shall confine myself to the enunciation of the general conclusions I have been able to ieduce, and to supporting them, as required, by several examples.

(I.) The centre of gravity in an insect is situated in the vertical and medial plane which passes along the longitudinal axis of the body.

(2.) It occupies a position almost identical in insects of the same species, the same sex, and in the same attitude.

(3.) The exterior form of the body rarely permits the determination of the exact position of the centre of gravity without experiment. I shall cite the results with which the family of Odonates have furnished me as examples. All its representatives have nearly the same exterior aspect; and yet, notwithstanding this quasi identity of structure, I have found in the relative position of the centre of gravity the following differences :-

Agrion puella, female Ist third of the $3^{\text {rd abdominal ring. }}$ " sanguinea ", Posterior border of the and abdominal ring.

Libellula conspurcata " " ", of metathorax. Libellula vulgata " " Groove between thorax and abAsschna grandis $\quad$, Middle of and abdominal ring.

(4.) The centre of gravity does not occupy the same position in the two sexes of one species. It is sometimes less and sometimes more to the rear in the females than in the males ; and its situation depends on the relations existing between the different dimensions of the individuals. One would suppose that the centre of gravity would always be situated further back in females than in males, as the abdomen of the former is in general more bulky than that of the males. During the metamorphosis from larva to perfect insect, the relative centre of gravity apVOL. v. proaches the head; the absolute centre, on the contrary, recedes from it. ${ }^{*}$ This apparent contradiction is very easily explained; the thorax of the larva is generally much re. duced, and the abdominal rings numerous. In the perfect insect the thorax has acquired considerable dimensions, and the number of abdominal rings has diminished. The thorax, prolonging itself more to the rear, has approached, so to speak, the centre of gravity, which also remains in the medial region of the body; and the abdomen shortening itself, the distance of its extremity from the point in question diminishes.

(5.) While standing, the centre of gravity is placed at the base of the abdomen, or in the posterior portion of the thorax, and usually in the centre of the length of the body.

(6.) When an insect is walking, its centre of gravity undergoes constant displacement about a mean point, but the distances of displacement are too small to be measured. In fact, if experiments are made with leaping Orthoptera, grass-hoppers, or Acridians, it is ascertained that the displacement of their enormous posterior members leads to changes in the situation of the centre of gravity, but these changes are so small that one arrives at the conclusion that it is impossible to measure them in ordinary insects.

(7.) The displacement of the centre of gravity, when the insect passes from the state of repose to that of flight, cannot be ascertained except with those species where the wings lie folded on the back when in a state of repose. The displacement is horizontal and from back to front.

For example, in the following species the displacement is :-

Dytiscus dimidiatus Hydrophilus piceus Melolontha vulgaris Notonecta glauca

Locusta viridissima Vespa vulgaris Plusia gamma
Eristalis tenax

(8.) During active flight the centre of gravity oscillates continually about a mean position, which corresponds with the instants when the extremities of the wings pass the point of crossing of the 8-shaped curve which they describe in the air.

(9.) In aquatic insects the centre of gravity is nearer to the lower than to the upper surface of the body.

(Io.) During swimming, the movements of the posterior feet, acting like oars, determine the oscillation of the centre of gravity around a mean position, which answers to the position of the swimming feet placed at the middle of their course. These oscillations of the centre of gravity lead to a continual swaying of the body about a transverse axis passing through the mean centre of gravity, and it ought, consequently, to follow a gently undulating course. Felix Plateau

* In my work 1 have called the relacive position of the centre of gravity, its position as regards any portion of the body, as rings, hip (hanche), \&c. : and I have named the absolute position of the centre of gravity the number which is obtained by calculating the relation between the distance of the centre of gravity from the posterior extremity of the body and the total length of the animal. The quotients, 050,067 , for example, obtained in this manner, mean that the distance of the centre of gravity from the posterior extremity is $x^{5}$ or Io $^{5}$ of the total length of the body. They show inmediately, and independently of the form and thinness of the rings, whether the to the anal orifice. 